\title{
EVALUACIÓN MICROBIOLÓGICA DE ALIMENTOS ADQUIRIDOS EN LA VÍA PÚBLICA EN UN SECTOR DEL NORTE DE BOGOTÁ
}

\section{MICROBIOLOGICAL EVALUATION OF FOOD ACQUIRED IN STREETS OF A NORTHERN AREA OF BOGOTÁ}

\author{
Martín A. Bayona R. ${ }^{1}$ \\ ${ }^{1}$ Bacteriólogo,' Esp., M.Sc. Facultad de Medicina, Universidad de Ciencias Aplicadas y Ambientales U.D.C.A. Calle 222 N ${ }^{\circ}$ \\ 55-37 Bogotá, D.C. E-mail: mabayona@udca.edu.co
}

Rev. U.D.C.A Act. E Div. Cient. 12 (2): 9-17, 2009

\section{RESUMEN}

Las enfermedades transmitidas por alimentos constituyen, a nivel mundial, uno de los problemas más generalizados y de mayor repercusión sobre la salud de personas, afectando generalmente a la población de bajos recursos, niños, mujeres embarazadas y ancianos. El presente trabajo permitió obtener información sobre la presencia de carga microbiana patógena (Salmonella sp. y Escherichia coli), en alimentos vendidos en la vía pública de un sector del norte de Bogotá (Colombia). Los alimentos evaluados correspondieron a arepa de maíz, perros calientes, hamburguesas, empanadas, chorizos, jugo natural de naranja, ensalada de frutas y pelanga, los cuales, fueron adquiridos a partir de 15 ventas ambulantes, durante doce semanas consecutivas, entre febrero y mayo de 2008. Se realizaron ensayos microbiológicos de ausencia-presencia, obteniendo un $11,8 \%$ y $25 \%$ de Salmonella spp. y E. coli, respectivamente, siendo evidente el riesgo microbiológico de los alimentos vendidos de esta manera.

Palabras clave: Salmonella spp., Escherichia coli, puestos callejeros de alimentos.

\section{SUMMARY}

Diseases passed on by food, constitute one of the most generalized problems and of mayor repercussion on human health, affecting normally the most vulnerable population, children, pregnant women and old aged people. This research intended to obtain information on the presence of microbiological charge (Salmonella spp. and Escherichia coli), present in food sold in streets of a northern area of Bogotá (Colombia), having in mind contributing factors, associated to the risk of microbial transmission. The food evaluated was represented by cornbread, hotdogs, hamburgers, empanadas, sausages, natural orange juice, fruit salad and "pelanga", obtained from 15 ambulatory vents during twelve consecutive weeks, between February and May, 2008. Absence-presence microbiological tests were carried out and an $8 \%$ and a $25 \%$ of Salmonella spp. and E. coli, respectively, was obtained, being the microbiological risk evidence evident of food sold in the streets.

Key words: Salmonella spp., Escherichia coli, street food stands.

\section{INTRODUCCIÓN}

Las enfermedades transmitidas por alimentos son el resultado de una amplia variedad de productos comestibles contaminados por microorganismos patógenos, toxinas o sustancias químicas. La prevención de las enfermedades de transmisión alimentaria depende de la manipulación cuidadosa de los productos crudos y de los productos terminados en la cadena de producción. 
Una óptima calidad y supervisión de los alimentos, se traduce en un ahorro importante de costos sociales, individuales de los consumidores y de los dueños de las industrias que los producen. Garantizar alimentos inocuos y de calidad ha sido una preocupación constante de quienes intervienen en una cadena de alimentos (Vargas et al. 2004; Fuentes et al. 2005). En Colombia, durante el año 2007, fueron reportados al sistema Nacional de Vigilancia en Salud Pública (SIVIGILA) 5563 casos de enfermedades transmitidas por alimentos y de los únicos cinco brotes con seguimiento, cuatro ocurrieron en restaurantes y uno en el hogar resaltando la importancia y la necesidad de mejorar los controles y vigilancia sobre los diferentes manipuladores (Instituto Nacional de Salud, 2008).

Una gran cantidad de la población Bogotana se ve en la necesidad de tomar los servicios de alimentación fuera del hogar, acudiendo a restaurantes y puestos callejeros de ventas de alimentos, donde se ingieren diferentes productos, sin que se guarden los elementales cuidados y procedimientos que se deben tener para la elaboración, la preparación, el consumo y la distribución de acuerdo con lo estipulado en la ley 09/79 y el decreto 3075/97. El proyecto de acuerdo 160 de 2004 del Concejo de Bogotá, establece los lineamientos generales para las actividades de control que ejerce la Secretaría Distrital de Salud, responsable de la vigilancia epidemiológica y Bioseguridad alimentaria, con el fin de prevenir eventos de intoxicación con alimentos (Alcaldía de Bogotá, 2004).

La inocuidad y la calidad de los productos comestibles constituye, conjuntamente con su disponibilidad y el acceso de los consumidores a estos y la estabilidad, uno de los pilares más relevantes de la seguridad alimentaria. La venta de alimentos en la vía pública es un fenómeno de gran importancia sanitaria, económica y sociocultural (Durango et al. 2004). Las características culturales y pésimas condiciones de higiene generan factores de riesgo potencial para la salud. En Colombia, las labores de manufactura de los alimentos vendidos en la vía pública, se realizan con poco o sin ningún tipo de control, en donde se incrementa la contaminación de los alimentos por la presencia de sustancias tóxicas inherentes a ellos, como en el caso de algunos hongos y crustáceos, por compuestos metálicos presentes en los empaques o equipos empleados en la preparación de alimentos y por la contaminación con productos químicos como plaguicidas (rodenticidas, fungicidas, herbicida e insecticidas), que llegan de una forma accidental. De igual manera, estos alimentos son preparados por personas sin la capacitación adecuada para su correcta manipulación, lo cual, produce contaminación microbiana, especialmente debida a bacterias, hongos, virus y parásitos. Además, puede ser preocupante el empleo de aditivos y de colorantes, la sensibilidad de las personas frente a algunos componentes de los alimentos y trastornos por la edad, como ocurre con la intolerancia a algunos alimentos por insuficiencias enzimáticas (Roberts et al. 2000; Vásquez et al. 2007).

Al observar la alta frecuencia de personas asociadas con la mala calidad microbiológica de los alimentos son múltiples los factores que contribuyen a los brotes de intoxicaciones, debidos a la ingestión de los mismos, donde se destacan varios factores, como la refrigeración inadecuada, la preparación de los alimentos con mucho tiempo de antelación al servicio, la cocción y el recalentamiento inapropiado. Además, influye el inadecuado aseo de los manipuladores, la obtención de alimentos a partir de fuentes contaminadas, la limpieza y la desinfección inadecuada de equipos y materiales empleados en la preparación de los alimentos y, finalmente, la localización de expendio en sitios inapropiados. Otros hallazgos, como presencia de insectos, ubicación y ventilación, se constituyen en factores determinantes que generan un escenario propicio para la contaminación y la propagación de microorganismos en alimentos (Merino, 2008; Mead et al. 1999).

Salmonella spp. y Escherichia coli son dos microorganismos asociados entre otras patologías, con la enfermedad diarreica aguda, la cual, es una de las causas más importantes de morbilidad y mortalidad sobre todo en lactantes, en niños y en adultos mayores (Fuentes et al. 2005; Rodríguez E Prado, 2006).

Las especies de Salmonella corresponden a patógenos para humanos y animales, adquiridos por vía oral, causando enteritis, infección sistémica y fiebre entérica. Se asocian con la ingestión de alimentos preparados o manipulados inapropiadamente o contaminados con antelación (Rodríguez \& Prado, 2006). Los brotes de transmisión alimentaria por Salmonella, se producen después de la ingestión de aves, carne vacuna, huevos o productos lácteos. Los huevos con cáscara contaminada producen brotes de infecciones por Salmonella enteritidis. Mencionan varios autores, como Mossel et al. (2003) y 
Merino (2008), que los brotes también son causados por productos vegetales frescos, como melones, tomates, jugo de naranja no pasteurizado y alfalfa.

E. coli es un bacilo corto y móvil Gram negativo, que habita normalmente en el tracto gastrointestinal del hombre y de los animales de sangre caliente, desempañando un importante papel dentro de la carga microbiana normal y fisiología del intestino. Generalmente, este microorganismo suele ser inocuo, pero algunas cepas son causantes de gastroenteritis y otras enfermedades. Su patogenicidad es bien conocida y se ha asociado a diarrea, colitis hemorrágica, disentería, infecciones urinarias y meningitis entre otras patologías. De acuerdo a O'Brien et al. (1983), Slutskers et al. (1997) y Borbolla et al. (2004), las E. coli asociadas con diarrea han sido clasificadas, con base en criterios clínicos, epidemiológicos y moleculares en cinco grupos. Para la industria de alimentos, su búsqueda se interpreta como un indicador del estado higiénico de los alimentos. Teniendo en cuenta que se desconoce el peligro que puede presentar la venta callejera de alimentos sin mayores cuidados en su preparación y dispendio, se realizó este estudio para concientizar y conocer la carga microbiana patógena presente.

\section{MATERIALES Y MÉTODOS}

El presente estudio correspondió a un análisis descriptivo, longitudinal, completamente al azar, para lo cual, se realizaron muestreos aleatorios en 15 puntos de venta de alimentos ambulantes, situados entre las calles 170 y 222 del norte de Bogotá, abarcando barrios de estratos uno al cuatro. Las muestras fueron compradas durante doce semanas consecutivas, entre febrero y mayo de 2008. Dentro de los alimentos evaluados en la búsqueda de Salmonella spp. y E. coli, figuraban arepa de maíz, perros calientes, hamburguesas, empanadas, chorizos crudos y cocinados, ensalada de frutas, jugo de naranja y pelanga.

Las muestras de los diferentes productos terminados fueron recogidas en envases y en bolsas estériles, escogiéndolos de manera aleatoria y representativa, para lo cual, se tomaron 68 muestras $(200 \mathrm{~g} \mathrm{y} / \mathrm{o} \mathrm{mL}$, aproximadamente, por alimento o bebida) (Tabla 1). Una vez identificadas y rotuladas, se transportaron en neveras portátiles al laboratorio de Microbiología de la Universidad de Ciencias Aplicadas y Ambientales U.D.C.A, donde se conservaron en refrigeración entre $0-4^{\circ} \mathrm{C}$ hasta el momento del análisis (Mossel et al. 2003).

En cada uno de los sitios de muestreo, se llevó a cabo una inspección con base en la observación y la entrevista con el vendedor. Dentro de los aspectos indagados, se tuvo en cuenta el empleo de agua potable, la calidad y la higiene de los utensilios, el área de preparación de los alimentos, la disposición higiénica de basuras, la conservación de los alimentos y los conocimientos básicos sobre la manipulación adecuada de alimentos.

Tabla 1. Clasificación de los alimentos evaluados en la vía pública en un sector del norte de Bogotá, D.C. durante los meses de febrero-mayo de 2008.

\begin{tabular}{|c|c|c|}
\hline Clasificación & Alimento & $N^{0}$ de sitios y repeticiones \\
\hline Alimento consumo fresco & Ensalada de frutas & 1 sitio, 4 repeticiones \\
\hline Alimento consumo fresco & Jugo de naranja & 2 sitios, 8 repeticiones \\
\hline Alimento cocinado & Arepa de maíz & 2 sitios, 8 repeticiones \\
\hline Alimento cocinado & Perros calientes & 2 sitios, 4 repeticiones \\
\hline Alimento cocinado & Hamburguesas & 2 sitios, 8 repeticiones \\
\hline Alimento cocinado & Empanadas & 3 sitios, 12 repeticiones \\
\hline Alimento cocinado & Chorizos & 1 sitio, 8 repeticiones \\
\hline Alimento cocinado & Pelanga & 1 sitio, 8 repeticiones \\
\hline Alimento crudo & Chorizo & 1 sitio, 8 repeticiones \\
\hline
\end{tabular}


Tratamiento microbiológico: Se siguió el procedimiento de ausencia/presencia para los dos microorganismos. E. coli: se preparó un homogenizado, pesando $20 \mathrm{~g} /$ $\mathrm{mL}$ del alimento; se añadió $180 \mathrm{~mL}$ de solución salina estéril dentro de bolsas plásticas; luego, se realizaron dos diluciones seriadas con base diez; seguidamente, se sembraron en superficie $0,1 \mathrm{~mL}$ de las muestras obtenidas en cada una de las diluciones y se distribuyeron con asa de drigalsky en los medios de cultivo agar MacConkey y placas deshidratadas para E. coli / coniformes, que emplea Agar VRB modificado (violeta rojo bilis), con X-GAl. Estos medios de cultivo, se incubaron a $37^{\circ} \mathrm{C}$ durante 24 horas. Posteriormente, se realizó una evaluación macroscópica de las colonias características y se determinó la identificación de género y especie, mediante la técnica RapID TM ONE System, que es un micrométodo cualitativo que utiliza sustratos convencionales y cromogénicos para la identificación de microorganismos de importancia clínica, como enterobacterias y otros bacilos seleccionados Gram negativos y negativos a la oxidasa (Roberts et al. 2000; Vargas et al. 2004).

Salmonella: se pesaron $25 \mathrm{~g} / \mathrm{mL}$ del alimento y se inocularon en $225 \mathrm{~mL}$ de los medios de agua peptonada (1\%) y BHI (caldo infusión cerebro corazón), que fueron incubados a $37^{\circ} \mathrm{C}$ por un tiempo de 24 horas; a partir de allí, se inoculó $1 \mathrm{~mL}$ de cada muestra en $9 \mathrm{~mL}$ de caldo Rappaport y caldo tetrationato; seguidamente, se sub-cultivó en medios de Salmonella shigella, Hecktoen y XLD, las cuales, se incubaron a $37^{\circ} \mathrm{C}$ por un tiempo de 24 horas. Las pruebas características de posibles Salmonellas fueron identificadas por pruebas bioquímicas, siguiendo el paso anterior para $E$. coli (Roberts et al. 2000; Vargas et al. 2004; Yánez et al. 2008).

Análisis estadístico: Los datos así obtenidos se sometieron a un análisis estadístico con el paquete Statistix 7.0 ${ }^{\circledR}$ en el cual se incluyeron análisis de varianza de 1 vía, Wilcoxon - rank summary test, $\mathrm{T}$ pariada y diferencias mínimas de Tukey.

\section{RESULTADOS Y DISCUSIÓN}

A partir de las 68 muestras analizadas, se determinó un $11,8 \%$ y $25 \%$ de Salmonella spp. y E. coli, respectivamente. Los alimentos asociados con Salmonella spp correspondieron a: ensalada de frutas, hamburguesa, pelanga y chorizo crudo, mientras que en todos los nueve tipos de alimentos evaluados, se determinó la presencia de E. coli. (Tabla 2).

Fuentes et al. (2005) encontraron Salmonella sp. (3\%) a partir de ensaladas de frutas y no reportaron este microorganismo en otros alimentos, como queso

Tabla 2. Microorganismos identificados en diferentes alimentos vendidos en la vía pública en un sector del norte de Bogotá, D.C.

\begin{tabular}{|c|c|c|c|c|c|}
\hline Alimento & $\begin{array}{c}\text { Muestras } \\
\text { analizadas }\end{array}$ & $\begin{array}{c}\text { Muestras positivas } \\
\text { (Salmonella spp.) }\end{array}$ & $\begin{array}{c}\text { Porcentaje de Muestras } \\
\text { contaminados con } \\
\text { Salmonella spp. }\end{array}$ & $\begin{array}{c}\text { Muestras } \\
\text { positivas } \\
\text { (E. coli) }\end{array}$ & $\begin{array}{c}\text { Porcentaje de Muestras } \\
\text { contaminados con } \\
\text { E. coli }\end{array}$ \\
\hline Ensalada de frutas & 4 & 1 & 25,0 & 1 & 25,0 \\
\hline Jugo de naranja & 8 & 0 & 0,0 & 1 & 12,5 \\
\hline Arepa de maíz & 8 & 0 & 0,0 & 2 & 25,0 \\
\hline Perro caliente & 4 & 0 & 0,0 & 1 & 25,0 \\
\hline Hamburguesas & 8 & 1 & 12,5 & 3 & 12,5 \\
\hline Empanadas & 12 & 0 & 0,0 & 1 & 25,0 \\
\hline Chorizos cocinados & 8 & 0 & 0,0 & 2 & 12,5 \\
\hline Pelanga & 8 & 3 & 37,5 & 5 & 25,0 \\
\hline Chorizo crudo & 8 & 3 & 37,5 & 17 & 62,5 \\
\hline TOTAL & 68 & 8 & 11,8 & 25,0 \\
\hline
\end{tabular}


fresco, jugo de naranja natural y carne de hamburguesa. Los resultados anteriores, se atribuyen, posiblemente, a la frecuente manipulación a la que se someten los alimentos de consumo fresco durante su preparación, sumado a las deficientes prácticas higiénicas y de almacenamiento que se desarrollan en estos sitios, lo cual, se pudo observar en el presente trabajo.

Los resultados obtenidos con este estudio son concordantes con los reportados por Durango et al. (2004), para el Caribe colombiano, en los que, se aislaron Salmonella spp., en un 7,4 \% de las muestras analizadas. Por tipo de alimento, se encontró dicho microorganismo en muestras de carne de res, en un $9,3 \%$, un $12,6 \%$ para chorizo, en un $7,9 \%$ de queso, en $5,2 \%$ de carne de cerdo, en $1,6 \%$ de muestras de pollo y en el $10,5 \%$ de arepas de huevo. La arepa de huevo, el chorizo y la carne de res fueron los alimentos con mayor contaminación con Salmonella spp., que está relacionado, posiblemente, con la excesiva manipulación y la diversidad de ingredientes empleados en su preparación, sumado a las deficientes condiciones de almacenamiento. Según Caballero et al. (1998), al analizar alimentos vendidos en las calles de todas las provincias de Cuba, desde enero de 1995 hasta junio de 1997, un 3,5\% estaba contaminado con Salmonella spp. En el presente trabajo, se encontró el doble del porcentaje, lo que es muy preocupante y es indicativo de una deficiente higiene en el procesamiento y manipulación de alimentos vendidos en la vía pública.

Por otra parte, el actual estudio no identificó la presencia de estos patógenos en los manipuladores, frente a lo que no se pudo establecer una asociación propiamente dicha. La posibilidad de una contaminación fecal de los alimentos, a través de las manos de los manipuladores representa, un alto riesgo, de acuerdo con Valdivieso et al. (2006), quienes al evaluar la presencia de patógenos bacterianos en frotis de manos en manipuladores de alimentos de tres comedores públicos, en CumanaVenezuela, hallaron E. coli en un 35,46\%. Borbolla et al. (2004), al analizar diferentes alimentos vendidos en la vía pública en el estado de Tabasco México, obtuvieron muestras positivas para Salmonella spp., en un 0,44\%, mientras que $E$. coli estuvo presente en los alimentos analizados, con una frecuencia de 35,6\%.

Restrepo et al. (2007) y Mossel et al. (2003) afirman que la familia Enterobacteriacea, a la que pertenecen
Salmonella spp. y E. coli, representan un grupo heterogéneo de bacilos Gram negativos, en las que las infecciones se adquieren por contigüidad con el tracto gastrointestinal, en el caso de las infecciones genitourinarias, en gastroenteritis, la transmisión es oro-fecal e intervienen portadores humanos o animales con vectores inanimados, como el agua o los alimentos contaminados; las pobres condiciones sanitarias contribuyen a la alta frecuencia de gastroenteritis por enterobacterias, en muchas regiones del mundo.

Dentro de los diferentes puntos críticos encontrados se tienen (Tabla 3):

- Deficiencias en la manipulación e higiene de los alimentos, en lo que concierne con buenas prácticas de manufactura, destacando el manejo simultáneo de dinero y de alimentos, así como el uso de joyas (anillos, relojes, aretes) y empleo de barniz para uñas, para el caso de las mujeres. Solamente el $13 \%$ de las ventas cumple con buenas prácticas de manufactura.

- No se emplea un adecuado suministro y manejo del agua; el $87 \%$ de las ventas no cumplió con este requisito.

- Se emplea material desechable para servir los alimentos (33\%), el cual, es reutilizado mediante un lavado con agua almacenada en garrafones

- Los medios utilizados para proteger los alimentos expuestos para la venta varían desde vitrinas de vidrio con bombillo incorporado, empleo de telas de algodón y tapas plásticas. El 93\% de los puestos de venta callejera no cumplieron con un buen almacenamiento de los alimentos.

- Los puestos de venta presentaron un deficiente manejo de basuras, expuestas al medio ambiente, frecuentadas por moscas. Solo el $27 \%$ de los puestos de venta callejera cumplían con un buen manejo de basuras. De acuerdo con la Alcaldía de Bogotá (2004), la eliminación inadecuada de las aguas residuales y la basura derivadas de la venta callejera de alimentos incrementan el potencial de transmisión de enfermedades microbianas, en parte, al estimular la proliferación de insectos y roedores transmisores de patógenos entéricos.

- El $73 \%$ de los vendedores no poseen conocimientos básicos sobre los microorganismos patógenos que se pueden transmitir por los alimentos y las enfermedades asociadas a ellos. 
Tabla 3. Puntos críticos en ventas de comida callejera.

\begin{tabular}{|c|c|c|c|c|c|c|c|c|c|c|c|c|c|c|c|c|c|c|c|c|c|c|c|c|c|c|c|c|}
\hline \multirow{3}{*}{$\begin{array}{c}\text { Características } \\
\text { evaluadas }\end{array}$} & \multicolumn{28}{|c|}{ Puestos de venta } \\
\hline & 1 & & 2 & & 3 & & 4 & 5 & & 6 & & 7 & & 8 & 9 & & 10 & & 11 & & 12 & & 13 & 14 & & 15 & & \\
\hline & $\mathrm{sl}$ & NO & SI & NO & $\mathrm{SI} \mid \mathrm{NC}$ & 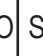 & $\mathrm{NC}$ & $\mathrm{SI}$ & NO & $\mathrm{SI} \mid \mathrm{N}$ & & $\mathrm{SI} \mid \mathrm{NC}$ & SI & NO & SI & NO & $\mathrm{SI} / \mathrm{NC}$ & $\mathrm{s}$ & NO & SI & NO & SI & NO & SI & NO & $\mathrm{SI} N$ & 0 & TIRL \\
\hline $\begin{array}{c}\text { Buenas prácticas } \\
\text { manufactura }\end{array}$ & $\mathrm{x}$ & & & $\mathrm{x}$ & $\mathrm{x}$ & & $\mathrm{x}$ & & $\mathrm{x}$ & $x$ & & $\mathrm{x}$ & & $\mathrm{x}$ & & $\mathrm{x}$ & $\mathrm{x}$ & & $\mathrm{x}$ & & $\mathrm{x}$ & & $\mathrm{x}$ & & $\mathrm{x}$ & $x$ & & $\begin{array}{l}\mathrm{Si}=13 \% / \\
\mathrm{NO}=87 \%\end{array}$ \\
\hline Suministros agua & $\mathrm{x}$ & & & $x$ & $\mathrm{x}$ & & $\mathrm{x}$ & & $x$ & $x$ & & $\mathrm{x}$ & & $\mathrm{x}$ & & $\mathrm{x}$ & $\mathrm{x}$ & & $\mathrm{x}$ & $x$ & & & $x$ & & $\mathrm{x}$ & $x$ & $x$ & $\begin{array}{c}\mathrm{Si}=13 \% \\
\mathrm{NO}=87 \%\end{array}$ \\
\hline $\begin{array}{l}\text { Material desechable } \\
\text { reutilizable }\end{array}$ & $\mathrm{x}$ & & $\mathrm{x}$ & & $\mathrm{x}$ & & $\mathrm{x}$ & & $\mathrm{x}$ & $x$ & & $x$ & & $\mathrm{x}$ & & $\mathrm{x}$ & $\mathrm{x}$ & & $x$ & & $\mathrm{x}$ & & $\mathrm{x}$ & $x$ & & $x$ & & $\begin{array}{c}\mathrm{Si}=33 \% \\
\mathrm{NO}=67 \%\end{array}$ \\
\hline $\begin{array}{l}\text { Mantenimiento y } \\
\text { conservación }\end{array}$ & & $\mathrm{x}$ & & $\mathrm{x}$ & $\mathrm{x}$ & & $\mathrm{x}$ & & $\mathrm{x}$ & $x$ & & $\mathrm{x}$ & & $\mathrm{x}$ & & $\mathrm{x}$ & $\mathrm{x}$ & & $\mathrm{x}$ & & $\mathrm{x}$ & & $\mathrm{x}$ & $\mathrm{x}$ & & $x x x$ & & $\begin{aligned} \mathrm{Si} & =7 \% \\
\mathrm{NO} & =93 \%\end{aligned}$ \\
\hline Manejo basuras & & $x$ & & $x$ & $\mathrm{x}$ & & $\mathrm{x}$ & & $\mathrm{x}$ & $x$ & & $\mathrm{x}$ & & $\mathrm{x}$ & & $\mathrm{x}$ & $\mathrm{x}$ & & $\mathrm{x}$ & $x$ & & $\mathrm{x}$ & & $x$ & & $\mathrm{x}$ & & $\begin{array}{c}\mathrm{Si}=27 \% \\
\mathrm{NO}=73 \%\end{array}$ \\
\hline $\begin{array}{c}\text { Conocimiento básico } \\
\text { manejo alimentos }\end{array}$ & & $\mathrm{x}$ & & $\mathrm{x}$ & $\mathrm{x}$ & & $\mathrm{x}$ & & $\mathrm{x}$ & $x$ & & $\mathrm{x}$ & & $\mathrm{x}$ & & $\mathrm{x}$ & $\mathrm{x}$ & & $\mathrm{x}$ & & $\mathrm{x}$ & $x$ & & $x$ & & $x$ & & $\begin{array}{c}\mathrm{Si}=20 \% \\
\mathrm{NO}=80 \%\end{array}$ \\
\hline
\end{tabular}

Una calidad garantizada del alimento, se traduce en un ahorro importante de costos sociales, individuales de los consumidores y de los dueños de las industrias que los producen. De acuerdo con Flórez et al. (2008), al determinar los factores relacionados con enfermedades transmitidas por alimentos en restaurantes de cinco ciudades de Colombia, encontraron importantes deficiencias en el cumplimiento de las buenas prácticas de manufactura, en los hábitos de los manipuladores y en las condiciones sanitarias de los expendios de alimentos, hallazgos que requieren la capacitación de los manipuladores en estos aspectos y fortalecer, desde la autoridad sanitaria, la inspección adecuada de las condiciones de calidad de los establecimientos y de las prácticas higiénicas y la vigilancia de las enfermedades transmitidas por alimentos, para prevenirlas en la población.

Roberts et al. (2000) manifiestan que las salsas (mayonesa, salsa tártara y salsa de tomate), empleadas como aderezos de hamburguesas y perros calientes, contienen, entre otros ingredientes, huevos, leche y/o crema, los cuales, pueden ser una fuente de Salmonella, que durante su preparación pueden ser contaminados por otros microorganismos. Estos productos contienen otros ingredientes, como el vinagre (ácido acético) y el zumo de limón (ácido cítrico), con un pH menor de 4,5, que evita la multiplicación de las bacterias causantes de toxi-infecciones alimentarias, pero permiten el crecimiento de mohos y de levaduras.

En el caso del jugo de naranja, difícilmente constituyen un peligro para la salud, debido a su $\mathrm{pH}$ ácido, aunque se ha visto que la Salmonella y coliformes pueden tolerar estas condiciones (Mossel et al. 2003).

La carne de bovino picada o molida, en particular la de hamburguesa, insuficientemente cocinada, puede ser una fuente importante de infección por $E$. coli y Salmonella spp. La carne cocinada se puede recontaminar a partir de las superficies y equipos durante su envasado y su manipulación (Salgado et al. 1999). En términos generales, se evidenció en el presente estudio $(p=0,0279)$, que los alimentos se contaminan independientemente con Salmonella sp.; por otra parte, se encontró que no hay dependencia de E. coli $(\mathrm{p}=0,0527)$ y Salmonella spp. $(\mathrm{p}=0,07)$ con los alimentos crudos y cocidos, como en el caso del chorizo. Basados en lo anterior y de acuerdo con la Alcaldía de Bogotá (2004), la educación de los manipuladores y de los consumidores constituye un complemento necesario. La educación busca sensibilizar 
a consumidores y manipuladores, hacer que tomen conciencia de su responsabilidad en la Calidad. Las condiciones actuales en América Latina, no muestran ningún indicio que puedan desaparecer las ventas de alimentos en la vía pública, antes, por el contrario, se va a disparar este tipo de prácticas.

Las investigaciones ecológicas han demostrado que $E$. coli proviene del tracto intestinal del hombre y de los animales de sangre caliente; su presencia indica que puede haber existido contaminación fecal y que el consumidor podría estar expuesto a patógenos entéricos cuando ingiera el alimento (Mossel et al. 2003; Forte et al. 2000).

De acuerdo con Rodríguez \& Prado (2006) y Salgado et al. (1999), las infecciones producidas por S. enteritidis han venido aumentando a nivel del mundo. Las salmonelas productoras de enteritis son organismos termótrofos, que crecen bien a $42^{\circ} \mathrm{C}$, pero no a temperaturas inferiores a $7^{\circ} \mathrm{C}$, por lo tanto, se debe evitar la contaminación cruzada con Salmonellas de los productos cocidos o cocinados a partir de alimentos crudos y del ambiente próximo a su conservación. La incidencia real de la salmonelosis todavía se desconoce en gran parte, ya que muchos brotes no se declaran a las autoridades de salud pública. Por otra parte, un hecho importante de la epidemiología de Salmonella es el predominio cosmopolita de relativamente pocos, de los aproximadamente 2200 serotipos conocidos.

Yánez et al. 2008, reportaron la presencia de Salmonella spp. en chorizo (12,3\%), empanada de carne $(4,1 \%)$ y queso $(5,3 \%)$ en contraste con la ausencia de este microorganismo en otros alimentos, como empanada de pollo y carne cocida, todo esto debido al alto grado de manipulación y a las malas condiciones en los sitios de expendio de cerdo y de carne molida.

Aunque el presente estudio no abarcó una extensa zona de la ciudad, los resultados muestran la presencia de microorganismos patógenos de mucha importancia en salud pública, comprometiéndonos a difundir dicha información, para así prevenir la enfermedad diarreica en la población vulnerable.

Según Salgado et al. (1999), el control de Salmonella, se debe basar en las óptimas medidas de higiene adoptadas en los mataderos, en la industria cárnica y en los diferentes servicios de alimentación, entre ellos, los alimentos preparados y vendidos en la vía pública.

Forte E Rebagliati (2000) mencionan que la higiene de las superficies, equipos y utensilios, representa uno de los pilares donde se asientan las buenas prácticas de manufactura, lo cual, es confirmado por Pérez et al. (1998) y por Quispe E Sánchez (2001), quienes manifiestan que la correcta higiene de los alimentos está determinada por diversos factores, entre los que se encuentran: condiciones de obtención de los mismos, características de los medios empleados para su transporte, temperaturas y condiciones de almacenamiento, estructura de los locales donde se manipulan los alimentos y las prácticas de los manipuladores.

Se concluye que la calidad microbiológica y sanitaria de los sitios evaluados constituye un problema potencial de salud para nuestra población expuesta día a día a estos alimentos. Además, se evidencian deficiencias referentes a las buenas prácticas de manufactura y la ausencia de programas de manejo de vectores mecánicos, como es el caso de la mosca.

La vigilancia de la comida vendida en la vía pública es un tema indiscutible que nos debe llevar a tomar conciencia del riesgo sanitario y de la importancia en la implementación de acciones correctivas así como en la capacitación y seguimiento de los manipuladores.

AGRADECIMIENTOS: El autor agradece la colaboración prestada por los directivos de la Facultad de Medicina de la U.D.C.A y al Dr. Andrés Felipe Muñoz por sus orientaciones y valiosos aportes con el programa estadístico. Conflicto de interés: El autor del presente artículo declara que no existe ningún conflicto de interés que ponga en riesgo la validez de los resultados presentados. Financiación: El trabajo fue financiado por la Facultad de Medicina de la Universidad de Ciencias Aplicadas y Ambientales U.D.C.A.

\section{BIBLIOGRAFÍA}

1. AlCALdíA DE BOGOTÁ D.C. 2004. Proyecto de acuerdo 160 de 2004.Concejo de Bogotá D.C. Disponible desde Internet en: http://www.alcaldiabogotá.gov.co/sisjur/normas/Norma1 (con acceso 02/27/09). 
2. BORBOLLA, M.; VIDAL, M.; PIÑA, O.; RAMÍREZ, I.; VIDAL, J. 2004. Contaminación de los alimentos por Vibrio cholerae, coliformes fecales, Salmonella, hongos, levaduras y Staphylococcus aureus en Tabasco durante 2003. Salud en Tabasco. 10(1-2):221-232.

3. CABALLERO, A.; CARRERA, J.; LENGOMÍN, F. 1998. Evaluación de la vigilancia microbiológica de alimentos que se venden en las calles. Rev. Cubana Aliment. Nutr. 12(1):7-10.

4. DURANGO, J.; ARRIETA, G.; MATTAR, S. 2004. Presencia de Salmonella spp. en un área del Caribe Colombiano: un riesgo para la salud pública. Biomédica. 24:89-96.

5. FLÓREZ, A.; RINCÓN, C.; GARZÓN, P.; VARGAS, N.; ENRÍQUEZ, C. 2008. Factores relacionados con enfermedades transmitidas por alimentos en restaurantes de cinco ciudades de Colombia, 2007. Infectio (Colombia). 12(4):255-266.

6. FORTE, L.; REBAGLIATI, J. 2000. Control bacteriológico en plantas frigoríficas y conocimiento del fenómeno biopelícula. Bol. Alimentario. Edit. Aldo Marzochi. No 13. Buenos Aires, Argentina. 12p.

7. FUENTES, A.; CAMPAS, O.; MEZA, M. 2005. Calidad sanitaria de alimentos disponibles al público de ciudad de Obregón, Sonora, México. Rev. Salud Pública y Nutrición. 6(3):8-17.

8. INSTITUTO NACIONAL DE SALUD. 2008. Sistema de Vigilancia Epidemiológica (SIVIGILA). Colombia, 2007. Disponible desde Internet en: http://www. ins.gov.co (con acceso 01/03/08).

9. MÁTTAR, S.; DURANGO, J.; MORENO, C.; ARRIETA, G. 2003. Epidemiology of Salmonella strains food-related in the Caribbean area of Colombia. 103rd Meeting, American Society for Microbiology, May 18-22, 2003. Washington, D.C. p.142.

10. MEAD, P.; SLUTSKER, L.; DIETZ, V.; McCAIG, L.; BRESEE, J.; SHAPIRO, C.; GRIFFIN, P.; TAUXE, R. 1999. Food-related illness and death in the United States. Emerg. Infect. Dis. 5:607-625.
11. MERINO, L. 2008. Importancia de los vegetales que se consumen crudos en la transmisión de enfermedades de origen alimentario. Disponible desde Internet en: http://www.siicsalud.com/des/ des043/05504016.htlm (con acceso 01/03/08).

12. MOSSEL, D.; MORENO, B.; STRUIJK, C. 2003. Microbiología de los alimentos. Ed. Acribia, S.A. (España). 703p.

13. 0’BRIEN, A.; LIVELY, T.; CHEN, M.; ROTHMAN, S.; FORMAL, S. 1983 Escherichia coli 0 157:H7 strains associated with haemorragic colitis in the United States produce a Shigella dysenteriae I (Shiga) like cytotoxin. Lancet. I, p.702.

14. PÉREZ, M.; BELMONTE, S.; MARTÍNEZ, J. 1998. Estudio microbiológico de los alimentos elaborados en comedores colectivos de alto riesgo. Rev. Española de Salud Pública. 72:67-75.

15. QUISPE, J.; SÁNCHEZ, V. 2001. Evaluación microbiológica y sanitaria de puestos de venta ambulatoria de alimentos del distrito de comas, Lima-Perú. Rev. Perú.Med. Exp. Salud Pública. 18(1-2):27-32.

16. RESTREPO M., A.; DÍAZ C., F.; ESTRADA M., S.; FRANCO R., L.; JARAMILLO A., J.; MAESTRE B., A.; OSPINA O., S.; ROBLEDO R, C.; ROBLEDO R., J. 2007. Fundamentos básicos de Medicina. Microbiología de las infecciones humanas. Corporación para Investigaciones Biológicas (Colombia). 914p.

17. ROBERTS, D.; HOOPER, W.; GREENWOOD, M. 2000. Microbiología práctica de los alimentos. Ed. Acribia, S.A. (España). p.276.

18. RODRÍGUEZ C., J.; PRADO C, J. 2006. Microbiología: lo esencial y lo práctico. Organización Panamericana de la Salud. (E.U). 248p.

19. SALGADO, J.; JARAMILLO, C.; NUNES, F. 1999. Salmonella spp. en tres tipos de chorizos, como peligro dentro de un sistema de análisis de riesgo e identificación de puntos críticos de control (HACCP), en una empacadora de la ciudad de México. Vet. Mex. 30:157-165. 
20. SLUTSKER, L.; RIES, A.A.; GREENE, K.D.; WELLS, J.G.; HUTWAGNER, L.; GRIFFIN, P.M. 1997. Escherichia coli 0157:H7 DIARRHEA IN THE United Status: clinical and epidemiologic features. Ann. Intern. Med. 126(7):505-513.

21. VALDIVIESO, N.; VILLALOBOS, L.; MARTÍNEZ, R. 2006. Evaluación microbiológica en manipuladores de alimentos de tres comedores públicos en Cumana-Venezuela. Rev. Soc. Ven. Microbiol. 26(2):95-100.

22. VARGAS, J.; CLAVO, N.; MÁTTAR, S. 2004. Detección de Escherichia coli O157:H7 y Salmonella sp en cerdos del departamento de Córdoba. Rev. MVZ Córdoba. 9(1):386-392.
23. VÁSQUEZ, G.; GÓMEZ, E.; GAMBOA, E. 2007. Condiciones higiénico-sanitarias de los servicios de alimentación en instituciones infantiles del instituto Colombiano de Bienestar Familiar de Bucaramanga, Colombia. Rev. Cubana Aliment. Nutr. 17:23-33.

24. YÁNEZ, E.; MÁTTAR, S.; DURANGO, A. 2008. Determinación de Salmonella spp. por PCR en tiempo real y método convencional en canales de bovinos y en alimentos de la vía pública de Montería, Córdoba. Infectio. 12(4):246-253.

Recibido: Enero 26 de 2009

Aceptado: Septiembre 5 de 2009 